\title{
NONLINEAR VOLTERRA INTEGRAL EQUATIONS WITH POSITIVE DEFINITE KERNELS
}

\section{OLOF J. STAFFANS}

ABSTRACT. Conditions are given for the asymptotic stability of the solution of a nonlinear Volterra integro-differential equation with convolution kernel. These conditions are less restrictive than most of the known ones for this equation.

1. Introduction. We investigate the asymptotic behavior as $t \rightarrow \infty$ of bounded solutions of the Volterra integro-differential equation

$$
x^{\prime}(t)+\int_{0}^{t} a(t-\tau) g(x(\tau)) d \tau=f(t) \quad(0 \leq t<\infty),
$$

where $a, f$, and $g$ are prescribed, real valued functions. We first prove

Theorem 1. Let

$$
\begin{gathered}
a \in L^{1}(0, \infty), \\
\int_{0}^{\infty} \cos (\omega t) a(t) d t>0 \quad(-\infty<\omega<\infty), \\
g \in C(-\infty, \infty), \\
f \in L^{1}(0, \infty) .
\end{gathered}
$$

Let

$$
x \in L^{\infty}(0, \infty)
$$

be a (locally absolutely continuous) solution of $(1)$ on $[0, \infty)$. Then

$$
g(x(t))-0 \text { as } t \rightarrow \infty \text {. }
$$

It may be remarked that if, in addition to the hypothesis of Theorem 1, one assumes the growth condition

$$
\begin{gathered}
|g(x)| \leq K\left(1+\int_{0}^{x} g(\sigma) d \sigma\right) \quad(-\infty<x<\infty), \\
\quad \underset{x- \pm \infty}{\lim \sup _{0}} \int_{0}^{x} g(\sigma) d \sigma=\infty,
\end{gathered}
$$

Received by the editors May 22, 1974.

AMS (MOS) subject classifications (1970). Primary 45D05. 
where $K$ is some positive constant, then it is readily shown (cf. [10, p. 22]) that (1) has a solution, and that every solution of (1) is bounded on $0 \leq t<$ $\infty$. For this one does not need the strict in equality in (3).

The asymptotic stability of (1) has been investigated by a number of authors, see e.g. [2], [4], [6], [8], [9] and [10]. The hypotheses have always included (4), and, with the exception of [9], also (5) (sometimes, however, in addition to the function $f$ another term has been admitted on the right side of (1), behaving roughly like a constant). In [6] and [8] the key condition on the kemel is that $a \not \equiv(0)$ is nonincreasing, convex and sufficiently smooth, which implies (3) (see also [4]). This convexity assumption has been replaced in [2] and [10] by $\int_{0}^{\infty} \cos (\omega t) a(t) d t \geq \epsilon\left(1+\omega^{2}\right)^{-1}(-\infty<\omega<\infty)$ for some positive $\epsilon$, which of course also is stronger than (3). Not all of the references mentioned above require the integrability condition (2), and it can also be relaxed here as we show in $\$ 3$. The result in [9] does not fall under the scope of our work, as it replaces (5) by $f(t) \rightarrow 0$ as $t \rightarrow \infty$, but then it requires instead the additional assumption $x g(x)>0(x \neq 0)$, and also a moment condition on $a$.

Before continuing the discussion it is useful to see how the proof of Theorem 1 is reduced to two lemmas, which are of independent interest.

2. Proof of Theorem 1. Multiply (1) by $g(x(t))$ and integrate to obtain

$$
\begin{aligned}
G(x(T))-G(x(0))+ & \int_{0}^{T} g(x(t)) \int_{0}^{t} a(t-\tau) g(x(\tau)) d \tau d t \\
& =\int_{0}^{T} g(x(t)) f(t) d t \quad(T>0),
\end{aligned}
$$

where $G(x)=\int_{0}^{x} g(\sigma) d \sigma$. Define $\phi(t)=g(x(t))(0 \leq t<\infty)$. From (4) and (6) we see that $\phi$ is bounded, and so is $G(x(T))(0 \leq T<\infty)$. Together with (5) and (8) this gives

$$
\sup _{T>0} \int_{0}^{T} \phi(t) \int_{0}^{t} a(t-\tau) \phi(\tau) d \tau d t<\infty .
$$

The boundedness of $\phi$ together with (2) implies that the integral term in (1) is bounded, i.e. $x^{\prime} \in L^{\infty}(0, \infty)+L^{1}(0, \infty)$. It then follows that $x$ is uniformly continuous on $[0, \infty)$, and so by $(4)$ and $(6), \phi$ is uniformly continuous on $[0, \infty)$. Hence Theorem 1 is a simple consequence of

Lemma 1. Let a satisfy (2) and (3). Let $\phi$ be a bounded and uniformly continuous function on $[0, \infty)$, and suppose that (9) holds. Then $\phi(t) \rightarrow 0$ as $t \rightarrow \infty$.

However, Lemma 1 follows immediately from Lemma 2 below, combined 
with Pitt's form of Wiener's Tauberian theorem.

Lemma 2. Let $a \in L^{1}(0, \infty)$ satisfy

$$
\int_{0}^{\infty} \cos (\omega t) a(t) d t \geq 0 \quad(-\infty<\omega<\infty) .
$$

Let $\phi \in L^{\infty}(0, \infty)$, and suppose that (9) holds. Define $a(-t)=a(t) \quad(0<t<$ $\infty)$. Then

$$
\int_{0}^{\infty} a(t-\tau) \phi(\tau) d \tau \rightarrow 0 \text { as } t \rightarrow \infty
$$

The proof of Lemma 2 is given in $\$ 4$.

3. Kernels of strictly positive type. Using a technique similar to the one used by Halanay in [2] and later by MacCamy and Wong in [10] we can remove the integrability condition (2) in Theorem 1 (however, then the uniform continuity of $g(x(t))$ used in the reduction of the proof of Theorem 1 to Lemma 1 must be established separately). The kernel $a$ can also easily be replaced by a Borel measure. Our results can furthermore be extended to Volterra equations in arbitrary Hilbert spaces, and this leads to a generalization of MacCamy's and Wong's "weak stability principle" [10, p. 5]. For simplicity we discuss below only the scalar case with a locally integrable kernel.

Following Halanay [2] and MacCamy and Wong [10] we define (for functions $a \in L^{1}(0, T)$ for every $\left.T, 0<T<\infty\right)$ :

Definition 1. The function $a$ is of positive type iff for every $\phi \epsilon$ $C[0, \infty)$ and for every $T>0$

$$
\int_{0}^{T} \phi(t) \int_{0}^{t} a(t-\tau) \phi(\tau) d \tau d t \geq 0
$$

Definition 2. The function $a$ is of strongly positive type iff there exist $\epsilon, a>0$ such that the function $\psi$ defined by $\psi(t)=a(t)-\epsilon e^{-\alpha t}(0 \leq t<\infty)$ is of positive type.

If $a$ is of strongly positive type, and if (9) holds, then

$$
\sup _{T>0} \int_{0}^{T} \phi(t) \int_{0}^{t} e^{-\alpha(t-\tau)} \phi(\tau) d \tau d t<\infty,
$$

and this is used in [10] to prove that $\phi(t) \rightarrow 0$ as $t \rightarrow \infty$. Employing the more powerful Lemma 1 we can generalize the weak stability principle (adapted to $R^{1}$ ) as follows:

Definition 3. A function $a$ is of strictly positive type iff there exists a 
function $b \in L^{1}(0, \infty)$ satisfying $\int_{0}^{\infty} \cos (\omega t) b(t) d t>0 \quad(-\infty<\omega<\infty)$, such that $a-b$ is of positive type.

Lemma 3. Let a be of strictly positive type. Let $\phi$ be a bounded and uniformly continuous function on $[0, \infty)$, and suppose that (9) holds. Then $\phi(t) \rightarrow 0$ as $t \rightarrow \infty$ 。

(The weak stability principle in $R^{1}$ is essentially Lemma 3 with "strictly positive" replaced by "strongly positive".)

Proof of Lemma 3. Apply Lemma 1 with the function $a$ replaced by the function $b$, given in Definition 3.

A slightly simplified version of the arguments in $\$ 2$, replacing Lemma 1 by Lemma 3, now gives

Theorem 2. Let a be of strictly positive type, and suppose that (4) and (5) hold. Let $x$ be a bounded, uniformly continuous (and locally absolutely continuous) solution of (1) on $[0, \infty)$. Then $g(x(t)) \rightarrow 0$ as $t \rightarrow \infty$.

For a discussion on sufficient conditions for the uniform continuity of the solution $x$, see [12].

To see that strict positivity generalizes strong positivity, compute

$$
\int_{0}^{\infty} \cos (\omega t) e^{-\alpha t} d t=\alpha /\left(\omega^{2}+\alpha^{2}\right)>0 \quad(-\infty<\omega<\infty)
$$

Positivity in the sense of Definition 1 is essentially equivalent to $\int_{0}^{\infty} \cos (\omega t) a(t) d t \geq 0$ (cf. [12]; the integral can be interpreted as a generalized Fourier transform when $\left.a \notin L^{1}(0, \infty)\right)$. Hence the main advantage of the concept strict positivity, compared to strong positivity, is the removal of the rather restrictive growth condition

$$
\underset{\omega \rightarrow \infty}{\lim \inf } \omega^{2} \int_{0}^{\infty} \cos (\omega t) a(t) d t>0
$$

implied by Definition 2 and (11). One example where (12) is violated is the following: Let $a \in C^{2}[0, \infty)$, let $a, a^{\prime}, a^{\prime \prime} \in L^{1}(0, \infty)$, and let $a^{\prime}(0)=0$. Then $\lim _{\omega \rightarrow \infty} \omega^{2} \int_{0}^{\infty} \cos (\omega t) a(t) d t=0$, as one can show using two integrations by parts and the Riemann-Lebesgue lemma. In other words, the local property $a^{\prime}(0)=0$ together with a sufficient degree of smoothness and restricted growth prevents strong positivity. However, there also exist nonnegative, nonincreasing, convex kemels satisfying (3) which are not strongly positive (see [12]); such kernels are always strictly positive. A more complete discussion of this question, as well as of the questions of generalizing Defini- 
tion 3, and of other, equivalent, descriptions of strict positivity, will be given elsewhere.

Some other generalizations of Theorem 1 can be obtained by studying the asymptotic behavior of $g(x(t))$ when $a$ is of positive type, but not of strictly positive type. Here the main tool is Lemma 2 combined with certain Tauberian theorems. Special examples of this type (i.e. convex kernels) have been treated by Levin and Nohel [5] and by Hannsgen [4] using a different technique; some further results on the example in [5] can be found in [3, pp. 464-467], [7, p. 61] and [11, pp. 301-304]. We shall also return to this question elsewhere.

4. Proof of Lemma 2. Using the evenness of $a$ and an application of Fubini's theorem we get

$$
\int_{0}^{T} \phi(t) \int_{0}^{T} a(t-\tau) \phi(\tau) d \tau d t=2 \int_{0}^{T} \phi(t) \int_{0}^{t} a(t-\tau) \phi(\tau) d \tau d t
$$

Define $\phi_{T}(t)=\phi(t)(0 \leq t \leq T), \phi_{T}(t)=0 \quad(t>T$ or $t<0)$. Then (9) and (13) can be combined into

$$
\sup _{T>0} \int_{-\infty}^{\infty} \phi_{T}(t) \int_{-\infty}^{\infty} a(t-\tau) \phi_{T}(\tau) d \tau d t \stackrel{\text { def }}{=} K<\infty
$$

Since $a \in L^{1}(-\infty, \infty), \phi_{T} \in L^{2}(-\infty, \infty)$, and convolution with a $L^{1}(-\infty, \infty)$ function maps $L^{2}(-\infty, \infty)$ into itself, we can apply Parseval's identity. This gives

$$
\sup _{T>0} \int_{-\infty}^{\infty} \hat{a}(\omega)\left|\hat{\phi}_{T}(\omega)\right|^{2} d \omega=2 \pi K
$$

where

$$
\begin{gathered}
\hat{a}(\omega)=\int_{-\infty}^{\infty} e^{-i \omega t} a(t) d t=2 \int_{0}^{\infty} \cos (\omega t) a(t) d t \\
\hat{\phi}_{T}(\omega)=\int_{-\infty}^{\infty} e^{-i \omega t} \phi_{T}(t) d t=\int_{0}^{T} e^{-i \omega t} \phi(t) d t
\end{gathered}
$$

By (10), (15) and the fact that $a \in L^{1}(0, \infty)$,

$$
0 \leq \hat{a}(\omega) \leq 2 \int_{0}^{\infty}|a(t)| d t \stackrel{\text { def }}{=} \alpha \quad(-\infty<\omega<\infty) .
$$

Multiplying the integrand in (14) by $\hat{a}(\omega)$ we then get

$$
\sup _{T>0} \int_{-\infty}^{\infty}\left|\hat{a}(\omega) \hat{\phi}_{T}(\omega)\right|^{2} d \omega \leq 2 \pi K \alpha .
$$

Hence by Parseval's identity and the definition of $\phi_{T}$, 


$$
\sup _{T>0} \int_{-\infty}^{\infty}\left[\int_{0}^{T} a(t-\tau) \phi(\tau) d \tau\right]^{2} d t \leq K \alpha .
$$

Now use Fatou's lemma together with $a \in L^{1}(-\infty, \infty), \phi \in L^{\infty}(0, \infty)$ to get

$$
\int_{-\infty}^{\infty}\left[\int_{0}^{\infty} a(t-\tau) \phi(\tau) d \tau\right]^{2} d t \leq K \alpha .
$$

This means that

$$
\psi(t) \stackrel{\text { def }}{=} \int_{0}^{\infty} a(t-\tau) \phi(\tau) d \tau \in L^{2}(-\infty, \infty)
$$

Employing once more the facts that $a \in L^{1}(-\infty, \infty), \phi \in L^{\infty}(0, \infty)$ it is not difficult to prove that $\psi$ is uniformly continuous on $(-\infty, \infty)$. Combining this with (16) we then get $\psi(t) \rightarrow 0$ as $t \rightarrow \infty$, and the proof of Lemma 2 is complete.

Acknowledgments. The author wishes to thank Professors D. F. Shea and J. A. Nohel for their many helpful suggestions.

\section{REFERENCES}

1. C. Corduneanu, Sur une équation intégrale de la theorie du réglage automatique, C. R. Acad. Sci. Paris 256 (1963), 3564-3567. MR 27 \#100.

2. A. Halanay, On the asymptotic behavior of the solutions of an integro-differential equation, J. Math. Anal. Appl. 10 (1965), 319-324. MR 31 \#579.

3. J. K. Hale, Sufficient conditions for stability and instability of autonomous functional-differential equations, J. Differential Equations 1 (1965), 452-482. MR 32 \# 1414.

4. K. B. Hannsgen, On a nonlinear Volterra equation, Michigan Math. J. 16 (1969), 365-376. MR 40 \#3225.

5. J. J. Levin and J. A. Nohel, On a nonlinear delay equation, J. Math. Anal. Appl. 8 (1964), 31-44. MR 29 \#445.

6. - Perturbations of a nonlinear.Volterra equation, Michigan Math. J. 12 (1965), 431-447. MR $32 \# 336$.

7. J. J. Levin and D. F. Shea, On the asymptotic behavior of the bounded solutions of some integral equations. I, II, III, J. Math. Anal. Appl. 37 (1972), 42-82; 288-326; 537-575. MR $46 \# 5971$.

8. S.-O. Londen, The qualitative behavior of the solutions of a nonlinear Volterra equation, Michigan Math. J. 18 (1971), 321-330. MR 45 \#2431.

9. - On the variation of the solutions of a nonlinear integral equation, J. Math. Anal. Appl. (to appear).

10. R. C. MacCamy and-J. S. W. Wong, Stability theorems for some functional equations, Trans. Amer. Math. Soc. 164 (1972), 1-37. MR 45 \#2432.

11. R. K. Miller, Asymptotic behavior of nonlinear delay-differential equations, J. Differential Equations 1 (1965), 293-305. MR 31 \#2521.

12. J. A. Nohel and D. F. Shea, Frequency domain methods for Volterra equations, Advances in Math. (to appear).

INSTITUTE OF MATHEMATICS, HELSINKI UNIVERSITY OF TECHNOLOGY, SF-02150 OTANIEMI, FINLAND 